\title{
Energy Conservation in the Thin Layer Approximation: IV. The Light Curve for Supernovae
}

\author{
Lorenzo Zaninetti \\ Physics Department, via P. Giuria 1, Turin, Italy \\ Email: zaninetti@ph.unito.it
}

How to cite this paper: Zaninetti, L. (2021) Energy Conservation in the Thin Layer Approximation: IV. The Light Curve for Supernovae. International Journal of Astronomy and Astrophysics, 11, 37-58. https://doi.org/10.4236/ijaa.2021.111003

Received: December 6, 2020

Accepted: February 6, 2021

Published: February 9, 2021

Copyright ( 2021 by author(s) and Scientific Research Publishing Inc. This work is licensed under the Creative Commons Attribution International License (CC BY 4.0).

http://creativecommons.org/licenses/by/4.0/

\begin{abstract}
The light curves (LC) for Supernova (SN) can be modeled adopting the conversion of the flux of kinetic energy into radiation. This conversion requires an analytical or a numerical law of motion for the expanding radius of the SN. In the framework of conservation of energy for the thin layer approximation, we present a classical trajectory based on a power law profile for the density, a relativistic trajectory based on the Navarro-Frenk-White profile for the density, and a relativistic trajectory based on a power law behaviour for the swept mass. A detailed simulation of the LC requires the evaluation of the optical depth as a function of time. We modeled the LC of SN 1993J in different astronomical bands, the LC of GRB 050814 and the LC GRB 060729 in the $\mathrm{keV}$ region. The time dependence of the magnetic field of equipartition is derived from the theoretical formula for the luminosity.
\end{abstract}

\section{Keywords}

Supernovae: General, Supernovae: (Individual: SN1993j), Gamma-Ray Burst: (Individual: GRB 050814), Gamma-Ray Burst: (Individual: GRB 060729)

\section{Introduction}

The number of observational and theoretical analyses of the light curves (LCs) for supernovae (SN) has increased in recent years. We list some of the recent treatments. The LC of the type Ia supernova 2018oh has an unusual two-component shape [1], the radio LC of SN 1998bw shows a double-peak profile, possibly associated with density variations in the circumstellar medium [2], the R-band LCs of 265 SNs from the Palomar Transient Factory are followed and a model-independent LC template is built from this data-set [3], SN 2007D 
(which is a luminous type Ic supernova) has a narrow LC and high peak luminosity that were explored with a multi-band model [4]. Evolutionary models for the LC were introduced using the STELLA software application [5], the conversion of the kinetic energy of ejecta to radiation at the reverse and forward shocks was introduced in [6], the LC was modeled in the framework of the radioactive decay of ${ }^{56} \mathrm{Co},{ }^{57} \mathrm{Co}$ and ${ }^{55} \mathrm{Fe}[7]$, the cosmological importance of the LC was analysed by [8], and PS15dpn was a luminous rapidly rising Type Ibn SN which was modeled in the framework of the circumstellar interaction (CSI) model plus ${ }^{56} \mathrm{Ni}$ decay [9]. The previous papers leave a series of questions unanswered.

- Given the observational fact that the radius-time relation in young SNRs follows a power law, is it possible to find a theoretical law of motion in the framework of the classical energy conservation?

- Can we express the flux of kinetic energy in an analytical way in a medium which is characterized by a decreasing density?

- Can we parametrize the conversion of the analytical or numerical flux of kinetic energy into the observed luminosity?

- Can we model the double-peak profile for the LC in the framework of the temporal variations of the optical thickness?

- Can we apply the classical and relativistic approaches to the LC of SNs and Gamma Ray Bursts (GRBs)?

- Can we model the evolution of the magnetic field?

This paper is structured as follows. In Section 2 we explore the power law fit model. Section 3 reviews the classical and relativistic conversion of the flux of kinetic energy into luminosity. Section 4 presents some analytical results for a classical law of motion, Section 5 introduces two new relativistic equations of motion, Section 6 presents the simulation of the LC for one SN and two GRBs and Section 7 presents the temporal evolution of the magnetic field as well as some evaluations for the accelerating clouds due to the Fermi II acceleration mechanism.

\section{Preliminaries}

This section presents the analysed SN and GRB, introduces the adopted statistics, and reviews the power law model as a useful fit for the radius-time relation in SNs.

\subsection{The Analysed SN and GRB}

The first SN to be analysed is SN 1993J, for which the temporary radius of expansion has been measured for $\approx 10 \mathrm{yr}$ in the radio band [10] [11]. Here we processed for the case of SN 1993J the LC for the $R$ band as reported in Figure 5 in [12], the $V$ band for a short number of days, $\approx 63$ days, which shows an oscillating behaviour, see Figure 4 in [13], the luminosity of the $H-\alpha$ plotted with the $2.0-8.0 \mathrm{keV} \mathrm{LC}$ as reported in Figure 5 in [14] and the radio flux density at $15.2 \mathrm{GHz}$ as observed by the Ryle Telescope [15] with data available at http://www.mrao.cam.ac.uk/ dag/sn1993j.html. 
The second object to be analysed is GRB 050814 at $0.3-10 \mathrm{keV}$, which covers the time interval $\left[10^{-5}-3\right]$ days, see [16] with data available at https://www.swift.ac.uk/xrt live cat/150314.

The third object to be analysed is GRB 060729 observed by the Ultraviolet and Optical Telescope (UVOT) in the time interval $\left[10^{-2}-26\right]$ days, see Figure 1 in [17].

\subsection{The Statistics}

The adopted statistical parameters are the percent error, $\delta$, between the theoretical value and approximate value, and the merit function $\chi^{2}$ evaluated as

$$
\chi^{2}=\sum_{i=1}^{N}\left[\frac{y_{i, \text { theo }}-y_{i, o b s}}{\sigma_{i}}\right]^{2}
$$

where $y_{i, o b s}$ and $\sigma_{i}$ represent the observed value and its error at position $i$, $y_{i, \text { theo }}$ is the theoretical value at position $i$ and $N$ is the number of elements of the sample.

\subsection{The Power Law Model}

The equation for the expansion of a SN may be modeled by a power law

$$
r(t)=C t^{\alpha} \text { fit }
$$

where $r$ is the radius of the expansion, $t$ is the time, and $\alpha_{\text {fit }}$ is an exponent which can be found numerically. The velocity is

$$
v(t)=C t^{\alpha}{ }^{f i t^{-1}} \alpha_{\text {fit }} .
$$

As a practical example, the radius $(\mathrm{pc})$ time ( $\mathrm{yr}$ ) relation in $\mathrm{SN} 1993 \mathrm{~J}$ is

$$
r(t)=0.0155 \times t^{0.828} \mathrm{pc}
$$

when $0.49 \mathrm{yr}<t<10.58 \mathrm{yr}$, see also Table 1 .

\section{Luminosity}

In these subsections we analyse the classical and relativistic conversion of the flux of kinetic energy into luminosity. The absorption of the produced radiation is parametrized by the optical thickness.

\subsection{Conversion of Energy}

In the classical case, the rate of transfer of mechanical energy, $L_{m}$, is

$$
L_{m}(t)=\frac{1}{2} \rho(t) 4 \pi r(t)^{2} v(t)^{3},
$$

where $\rho(t), r(t)$ and $v(t)$ are the temporary density, radius and velocity of the $\mathrm{SN}$. We assume that the density in front of the advancing expansion scales as

$$
\rho(t)=\rho_{0}\left(\frac{r_{0}}{r(t)}\right)^{d},
$$


where $r_{0}$ is the radius at $t_{0}$ and $d$ is a parameter which allows matching the observations; as an example, a value of $d=3$ is reported in [18]. With the above assumption, the mechanical luminosity is

$$
L_{m}(t)=\frac{1}{2} \rho_{0}\left(\frac{r_{0}}{r(t)}\right)^{d} 4 \pi r(t)^{2} v(t)^{3} .
$$

The mechanical luminosity in the case of a power law dependence for the radius is

$$
L_{m}(t)=2 \rho_{0} r_{0}^{d} C_{f i t}^{-d+5} t^{-3+(-d+5) \alpha_{f i}} \pi \alpha_{f i t}^{3} .
$$

The energy fraction of the mechanical luminosity deposited in the frequency $v, L_{v}$, is assumed to be proportional to the mechanical luminosity through a constant $\epsilon_{v}$

$$
L_{v}=\epsilon_{v} L_{m} .
$$

The flux at frequency $v$ and distance $D$ is

$$
F_{v}=\frac{\epsilon_{v} L_{m}}{4 \pi D^{2}} .
$$

For practical purposes, we impose a match between the observed luminosity, $L_{o b s}$, and the theoretical luminosity, $L_{m}$,

$$
L_{o b s}=C_{o b s} L_{m} \text {, }
$$

where $C_{o b s}$ is a constant which equalizes the observed and the theoretical luminosity and varies on the base of the selected astronomical band. In a analogous way, the observed absolute magnitude is

$$
M_{\text {obs }}=-\log _{10}\left(L_{m}\right)+k_{\text {obs }},
$$

where $k_{o b s}$ is a constant. In the relativistic case the rate of transfer of mechanical energy, $L_{m, r}$, assuming the same scaling for the density in the advancing layer, is

$$
L_{m, r}(t)=4 \frac{\pi r(t)^{2} \rho_{0} c^{3} \beta(t)}{1-\beta(t)^{2}}\left(\frac{r_{0}}{r}\right)^{d},
$$

where $\beta(t)=\frac{v(t)}{c}$, for more details, see [19].

A useful formula is that for the minimum magnetic field density, $B_{\min }$,

$$
B_{\min }=1.8\left(\eta \frac{L_{v}}{V}\right)^{2 / 7} v^{1 / 7} T,
$$

where $v$ is the considered frequency of synchrotron emission, $L_{v}$ is the luminosity of the radio source at $v, V$ is the volume involved, and $\eta=\frac{\epsilon_{\text {total }}}{\epsilon_{e}}$ is a constant which connects the relativistic energy of the electrons, $\epsilon_{e}$, with the total energy in non-thermal phenomena, $\epsilon_{\text {total }}$, see formula (16.50) in [20] or formula (7.14) in [21]. 


\subsection{Absorption}

The presence of the absorption can be parametrized introducing a slab of optical thickness $\tau_{v}$. The emergent intensity $I_{v}$ after the entire slab is

$$
I_{v}=\int_{0}^{\tau_{v}} S_{v} \mathrm{e}^{-t} \mathrm{~d} t
$$

where $S_{v}$ is a uniform source function. Integration gives

$$
I_{v}=S_{v}\left(1-\mathrm{e}^{-\tau_{v}}\right),
$$

see formula 1.30 in [22]. In the case of an optically thin medium, $\tau_{v}=\infty$, the observed luminosity can be derived with Equation (11), but otherwise, the following equation should be used:

$$
L_{o b s}=C_{o b s} L_{m}\left(1-\mathrm{e}^{-\tau_{v}}\right),
$$

where $\tau_{v}$ is a function of time. For the case of the apparent magnitude, we have

$$
m_{o b s}=-\log _{10}\left(L_{m}\right)-\log _{10}\left(1-\mathrm{e}^{-\tau_{v}}\right)+k_{o b s} .
$$

The value of $\tau_{v}$ can be derived with the following equation:

$$
\tau_{v}=-\ln \left(1-\mathrm{e}^{-\left(m_{\text {obs }}-m_{\text {theo }}\right) \ln (10)}\right)
$$

where $m_{\text {theo }}$ and $m_{\text {obs }}$ represent the theoretical and the observed apparent magnitude. Due to the complexity of the time dependence of $\tau_{v}$, a polynomial approximation of degree $M$ is used:

$$
\tau_{v}(t)=a_{0}+a_{1} t+a_{2} t^{2}+\cdots+a_{M} t^{M},
$$

with more details in [23]. In some cases we apply the logarithms to the pair of data, i.e. $\log _{10}\left(x_{i}\right)$ and $\log _{10}\left(y_{i}\right)$; we call this the logarithmic polynomial approximation.

The absorption in the relativistic case is assumed to be the same once the classical luminosity, $L_{m}$, is replaced by the relativistic luminosity $L_{m, r}$

$$
L_{o b s}=C_{o b s} L_{m, r}\left(1-\mathrm{e}^{-\tau_{v}}\right),
$$

and

$$
m_{o b s}=-\log _{10}\left(L_{m, r}\right)-\log _{10}\left(1-\mathrm{e}^{-\tau_{V}}\right)+k_{o b s} .
$$

\section{A Classical Equation of Motion}

Let us analyse the case of conservation of energy in the thin layer approximation in the presence of a power law profile of density of the type

$$
\rho\left(r ; r_{0}\right)= \begin{cases}\rho_{c} & \text { if } r \leq r_{0} \\ \rho_{c}\left(\frac{r_{0}}{r}\right)^{\alpha} & \text { if } r>r_{0}\end{cases}
$$

where $\rho_{c}$ is the density at $r=0, r_{0}$ is the radius after which the density starts to decrease and $\alpha>0$, see Section 3.5 of [24]. The asymptotic radius is 


$$
\begin{aligned}
r(t)= & 12^{(\alpha-5)^{-1}} r_{0}^{\frac{\alpha-3}{\alpha-5}}\left(-4 r_{0} v_{0}(\alpha-5)\left(t-t_{0}\right) \sqrt{9-3 \alpha}\right. \\
& \left.-(\alpha-3)(\alpha-5)^{2}\left(t-t_{0}\right)^{2} v_{0}^{2}+12 r_{0}^{2}\right)^{-(\alpha-5)^{-1}}
\end{aligned}
$$

and the asymptotic velocity

$$
\begin{aligned}
v(t)= & 2\left(-4 r_{0} v_{0}(\alpha-5)\left(t-t_{0}\right) \sqrt{9-3 \alpha}\right. \\
& \left.-(\alpha-3)(\alpha-5)^{2}\left(t-t_{0}\right)^{2} v_{0}^{2}+12 r_{0}^{2}\right)^{\frac{4-\alpha}{\alpha-5}} \\
& \times\left(2 r_{0}^{\frac{2 \alpha-8}{\alpha-5}} \sqrt{9-3 \alpha}+v_{0} r_{0}^{\frac{\alpha-3}{\alpha-5}}(\alpha-3)(\alpha-5)\left(t-t_{0}\right)\right) 12^{(\alpha-5)^{-1}} v_{0} .
\end{aligned}
$$

An example of trajectory is reported in Figure 1 with data as in Table 1.

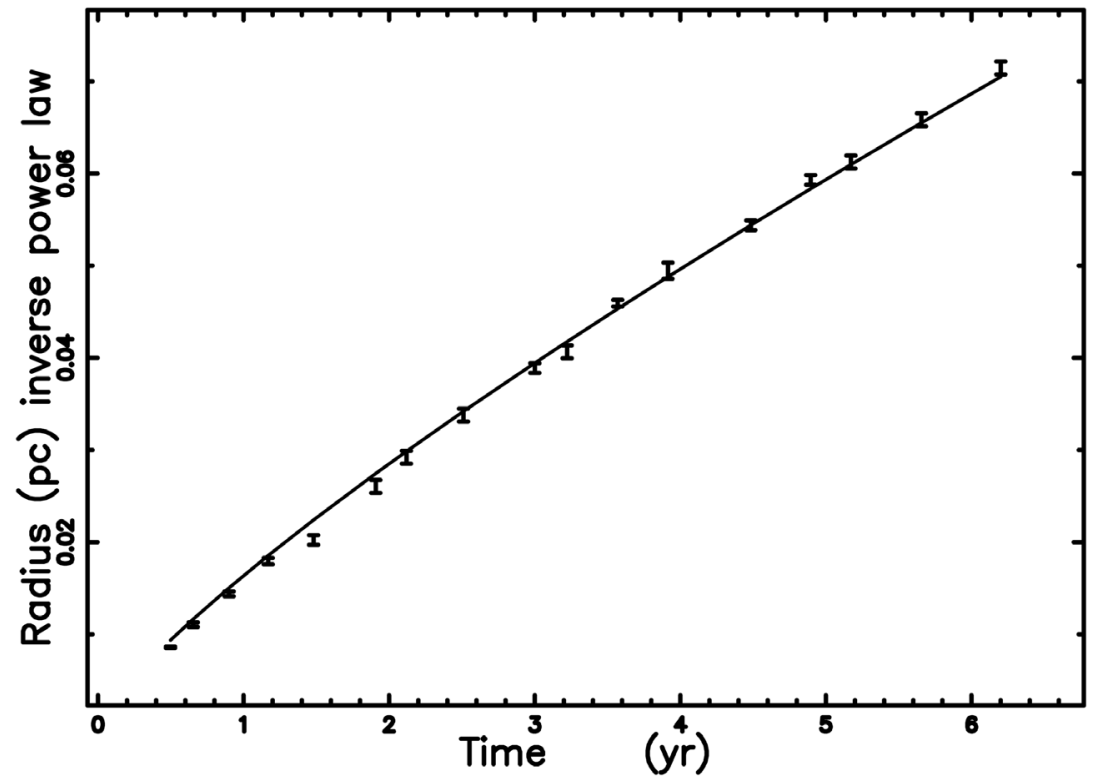

Figure 1. Theoretical radius as given by Equation (24), $v_{0}=4000 \mathrm{~km} / \mathrm{s}, t_{0}=10 \mathrm{yr}$ and $t=5 \times 10^{4} \mathrm{yr}$. The model is the conservation of the classical energy in the presence of an inverse power law profile for the density.

Table 1. Numerical values of the parameters for the fit and the theoretical models applied to SN 1993J.

\begin{tabular}{ccc}
\hline model & values & $\chi^{2}$ \\
\hline Fit by a power law & $\alpha_{\text {fit }}=0.828 ; C=0.015 ;$ & 43 \\
Classic power law profile & $\alpha=2.5 ; r_{0}=1.0 \times 10^{-5} \mathrm{pc} ;$ \\
$t_{0}=5 \times 10^{-4} \mathrm{yr} ; v_{0}=20000 \mathrm{~km} / \mathrm{s}$ & 176.6 \\
Relativistic NFW & $b=0.00185 \mathrm{pc} ; r_{0}=1 \times 10^{-4} \mathrm{pc} ;$ \\
& $t_{0}=3.6 \times 10^{-4} \mathrm{yr} ; v_{0}=269813 \mathrm{~km} / \mathrm{s}$ \\
Relativistic NCD & $\delta=1.16 ; r_{0}=5 \times 10^{-5} \mathrm{pc} ;$ \\
& $t_{0}=1.8 \times 10^{-4} \mathrm{yr} ; v_{0}=269813 \mathrm{~km} / \mathrm{s}$
\end{tabular}


As a consequence, we may derive an expression for the theoretical luminosity in presence of an inverse power law profile, $L_{\text {theo }}$, based on Equations (7) and (11)

$$
\begin{aligned}
L_{\text {theo }}= & \rho_{0} 128 r_{0}^{\frac{-2 d+5 \alpha-15}{\alpha-5}} v_{0}^{3} 12^{\frac{5-d}{\alpha-5}}\left(-4 r_{0} v_{0}(\alpha-5)\left(t-t_{0}\right) \sqrt{9-3 \alpha}\right. \\
& \left.-(\alpha-3)(\alpha-5)^{2}\left(t-t_{0}\right)^{2} v_{0}^{2}+12 r_{0}^{2}\right)^{\frac{d+10-3 \alpha}{\alpha-5}} \pi \\
& \times\left(r_{0} \sqrt{9-3 \alpha}+\frac{v_{0}(\alpha-3)(\alpha-5)\left(t-t_{0}\right)}{2}\right)^{3} .
\end{aligned}
$$

The above luminosity is based on theoretical arguments and no fitting procedure was used. The observed luminosity, $L_{o b s}$, can be obtained introducing

$$
L_{o b s}=C_{o b s} \times L_{\text {theo }} \text {, }
$$

where $C_{o b s}$ is a constant. Similarly,

$$
M_{\text {obs }}=-\log _{10}\left(L_{\text {theo }}\right)+k_{\text {obs }} \text {. }
$$

\section{The Relativistic Equation of Motion}

The relativistic conservation of kinetic energy in the thin layer approximation as derived in [25] is

$$
M_{0}\left(r_{0}\right) c^{2}\left(\gamma_{0}-1\right)=M(r) c^{2}(\gamma-1)
$$

where $M_{0}\left(r_{0}\right)$ and $M(r)$ are the swept masses at the two radii $r_{0}$ and $r$ respectively, $\gamma_{0}=\frac{1}{\sqrt{1-\beta_{0}^{2}}}$ and $\beta_{0}=\frac{v_{0}}{c}$.

\subsection{The NFW Profile}

We assume that the medium around the SN scales as the Navarro-Frenk-White (NFW) profile:

$$
\rho\left(r ; r_{0}, b\right)= \begin{cases}\rho_{c} & \text { if } r \leq r_{0} \\ \frac{\rho_{c} r_{0}\left(b+r_{0}\right)^{2}}{r(b+r)^{2}} & \text { if } r>r_{0}\end{cases}
$$

where $\rho_{c}$ is the density at $r=0$ and $r_{0}$ is the radius after which the density starts to decrease, see [26]. The total mass swept, $M\left(r ; r_{0}, b, \rho_{c}\right)$, in the interval $[0, r]$ is

$$
\begin{aligned}
M\left(r ; r_{0}, \rho_{c}, b\right)= & \frac{4 \rho_{c} \pi r_{0}^{3}}{3}+4 \frac{\rho_{c}\left(b+r_{0}\right)^{2}((b+r) \ln (b+r)+b) r_{0} \pi}{b+r} \\
& -4 \rho_{c}\left(b+r_{0}\right)\left(\left(b+r_{0}\right) \ln \left(b+r_{0}\right)+b\right) r_{0} \pi .
\end{aligned}
$$

Inserting the above mass in Equation (29) makes it possible to derive the velocity of the trajectory as a function of the radius as well as the differential equation of the first order which regulates the motion. The differential equation has a complicated behaviour which is not presented and Figure 2 displays the numerical 


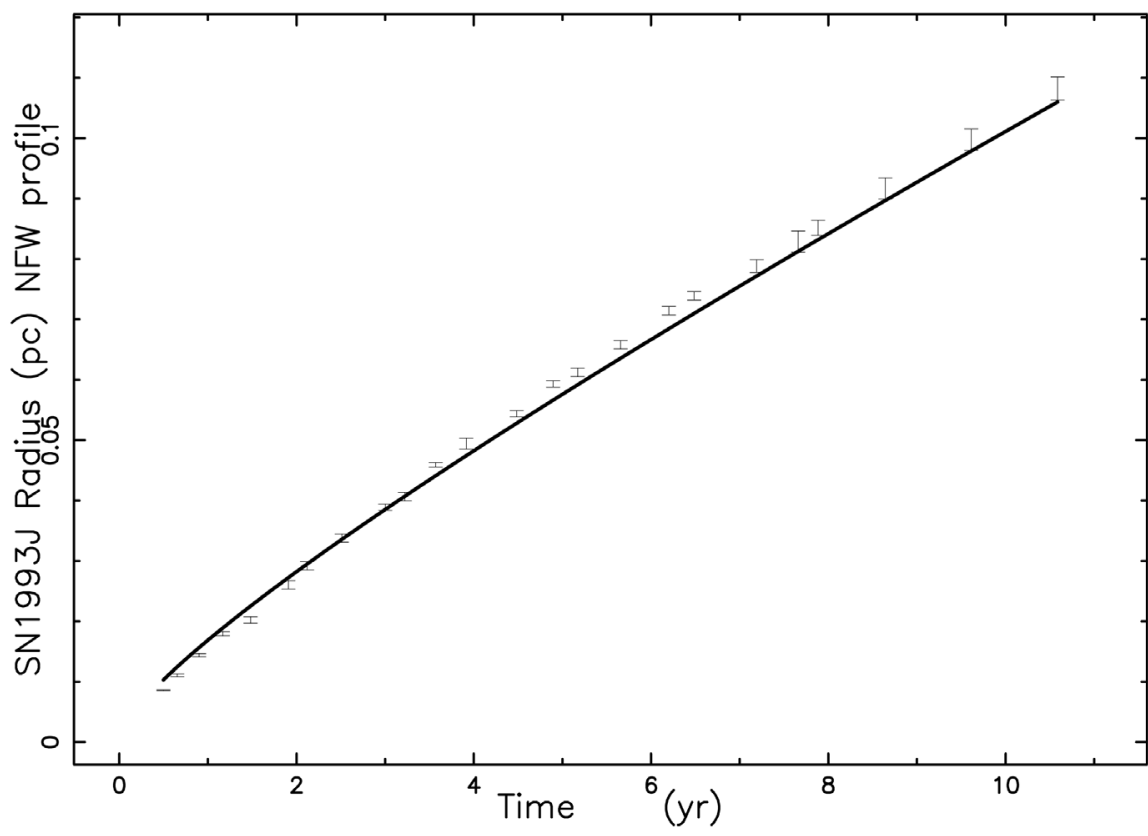

Figure 2. Numerical radius for the NFW profile (full line), with data as in Table 1. The model is the conservation of the relativistic energy in the presence of an NFW profile for the density.

solution.

Conversely, we present an approximate solution as a third-order Taylor series expansion about $r=r_{0}$

$$
\begin{aligned}
& r\left(t ; r_{0}, v_{0}, t_{0}, b\right) \\
& =\frac{1}{2 r_{0} c}\left(3\left(t-t_{0}\right)^{2}\left(-v_{0}+c\right)^{2}\left(v_{0}+c\right)^{2} \sqrt{\left(c^{2}-v_{0}^{2}\right)^{-1}}\right. \\
& \left.-3 c\left(\left(t-t_{0}\right)^{2} c^{2}-t_{0}^{2} v_{0}^{2}+\left(2 t v_{0}^{2}+2 / 3 r_{0} v_{0}\right) t_{0}-t^{2} v_{0}^{2}-2 / 3 t r_{0} v_{0}-2 / 3 r_{0}^{2}\right)\right) .
\end{aligned}
$$

Figure 3 presents the Taylor approximation of the trajectory in the restricted range of time $\left[4 \times 10^{-4} \mathrm{yr}-10^{-3} \mathrm{yr}\right]$.

\subsection{NCD Case}

We assume that the swept mass scales as

$$
M\left(r ; r_{0}, \delta\right)= \begin{cases}M_{0} & \text { if } r \leq r_{0} \\ M_{0}\left(\frac{r}{r_{0}}\right)^{\delta} & \text { if } r>r_{0}\end{cases}
$$

where $M_{0}$ is the swept mass at $r=0, r_{0}$ is the radius after which the swept mass starts to increase and $\delta$ is a regulating parameter less than 3. The differential equation of the first order which regulates the motion is obtained inserting the above $M(r)$ in Equation (29)

$$
\frac{\mathrm{d} r\left(t ; r_{0}, v_{0}, c, \delta\right)}{\mathrm{d} t}=\frac{A N}{A D}
$$




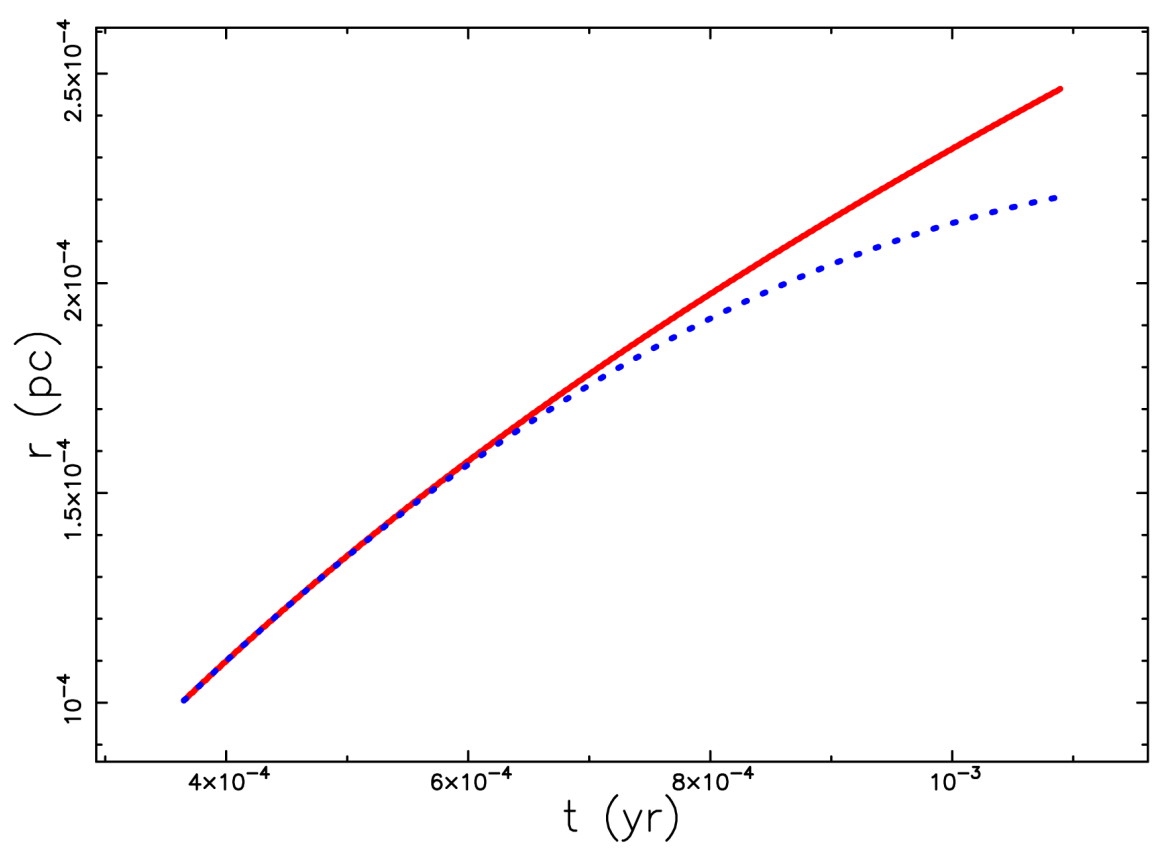

Figure 3. Numerical solution (full red line) and Taylor approximation (blue dashed line) for the NFW profile with parameters as in Table 1 . The model is the conservation of the relativistic energy in the presence of an NFW profile for the density.

where

$$
\begin{aligned}
A N= & \left(1 6 ( c - v _ { 0 } ) c \left(r_{0}^{-2 \delta}\left(-5 / 8 c^{2}+5 / 8 v_{0}^{2}\right)(r(t))^{2 \delta}\right.\right. \\
& +r_{0}^{-3 \delta}\left(1 / 8 c^{2}-1 / 8 v_{0}^{2}\right)(r(t))^{3 \delta}+(r(t))^{\delta}\left(c^{2}-3 / 4 v_{0}^{2}\right) r_{0}^{-2 \delta} \\
& \left.-1 / 2 c^{2}+1 / 4 v_{0}^{2}\right)\left(c+v_{0}\right) \sqrt{\left(c^{2}-v_{0}^{2}\right)^{-1}} \\
& +\left(10 c^{4}-15 c^{2} v_{0}^{2}+5 v_{0}^{4}\right) r_{0}^{-2 \delta}(r(t))^{2 \delta}-2 r_{0}^{-3 \delta}\left(c-v_{0}\right)^{2}\left(c+v_{0}\right)^{2}(r(t))^{3 \delta} \\
& \left.+\left(-16 c^{4}+20 c^{2} v_{0}^{2}-4 v_{0}^{4}\right)(r(t))^{\delta} r_{0}^{-\delta}+8 c^{4}-8 c^{2} v_{0}^{2}+v_{0}^{4}\right)^{1 / 2} c,
\end{aligned}
$$

and

$$
\begin{aligned}
A D= & 2 c\left(c-v_{0}\right)\left(c+v_{0}\right)\left(r_{0}^{-\delta}(r(t))^{\delta}-1\right) \sqrt{\left(c^{2}-v_{0}^{2}\right)^{-1}} \\
& +r_{0}^{-2 \delta}\left(c^{2}-v_{0}^{2}\right)(r(t))^{2 \delta}+\left(-2 c^{2}+2 v_{0}^{2}\right)(r(t))^{\delta} r_{0}^{-\delta}+2 c^{2}-v_{0}^{2} .
\end{aligned}
$$

The above differential does not have an analytical solution and therefore the solution should be derived numerically except about $r=r_{0}$ where a third-order Taylor series expansion gives

$$
\begin{aligned}
r\left(t ; r_{0}, v_{0}, t_{0}, \delta\right)= & r_{0}+v_{0}\left(t-t_{0}\right)+\frac{\delta\left(c-v_{0}\right)\left(c+v_{0}\right)\left(t-t_{0}\right)^{2}}{2 c r_{0}} \\
& \times\left(c^{2}-c \sqrt{c^{2}-v_{0}^{2}}-v_{0}^{2}\right) \frac{1}{\sqrt{c^{2}-v_{0}^{2}}}
\end{aligned}
$$

Figure 4 presents the numerical solution and Figure 5 the Taylor approximation of the trajectory. 


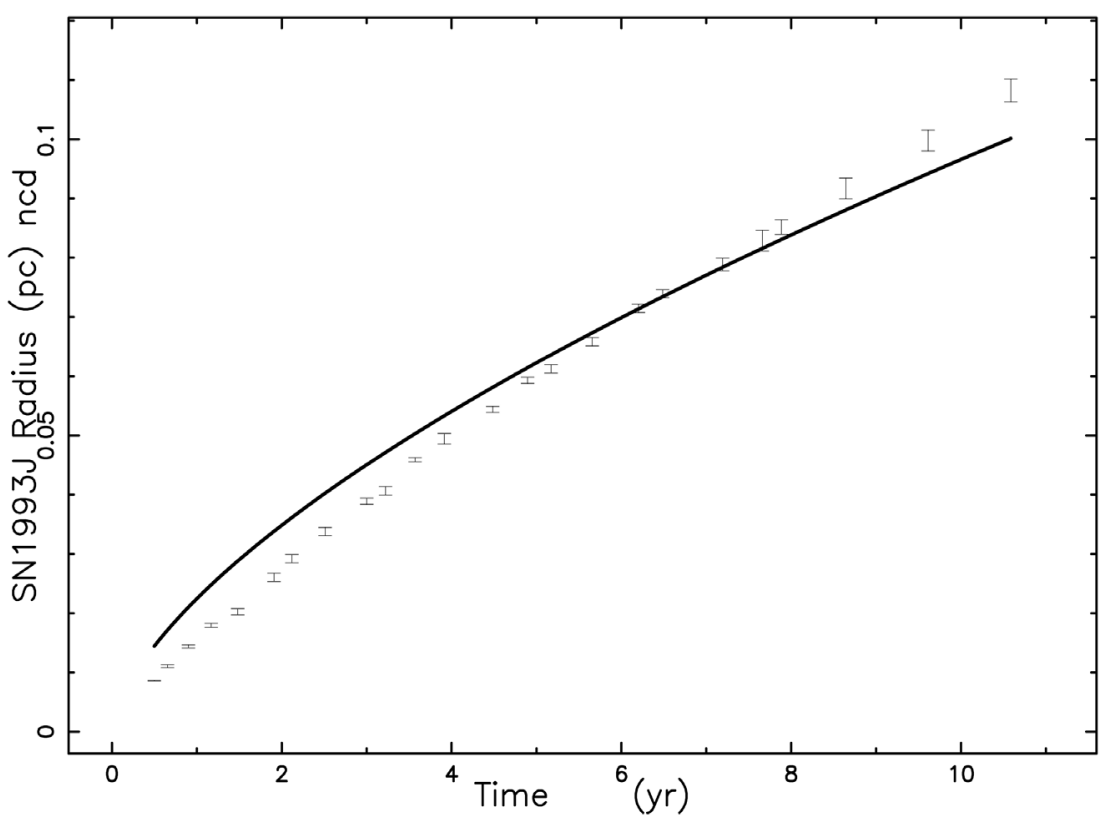

Figure 4. Numerical solution of the differential Equation (34) for the NCD case (full line), with data as in Table 1. The astronomical data of SN 1993J are represented with vertical error bars. The model is the conservation of the relativistic energy in the NCD case.

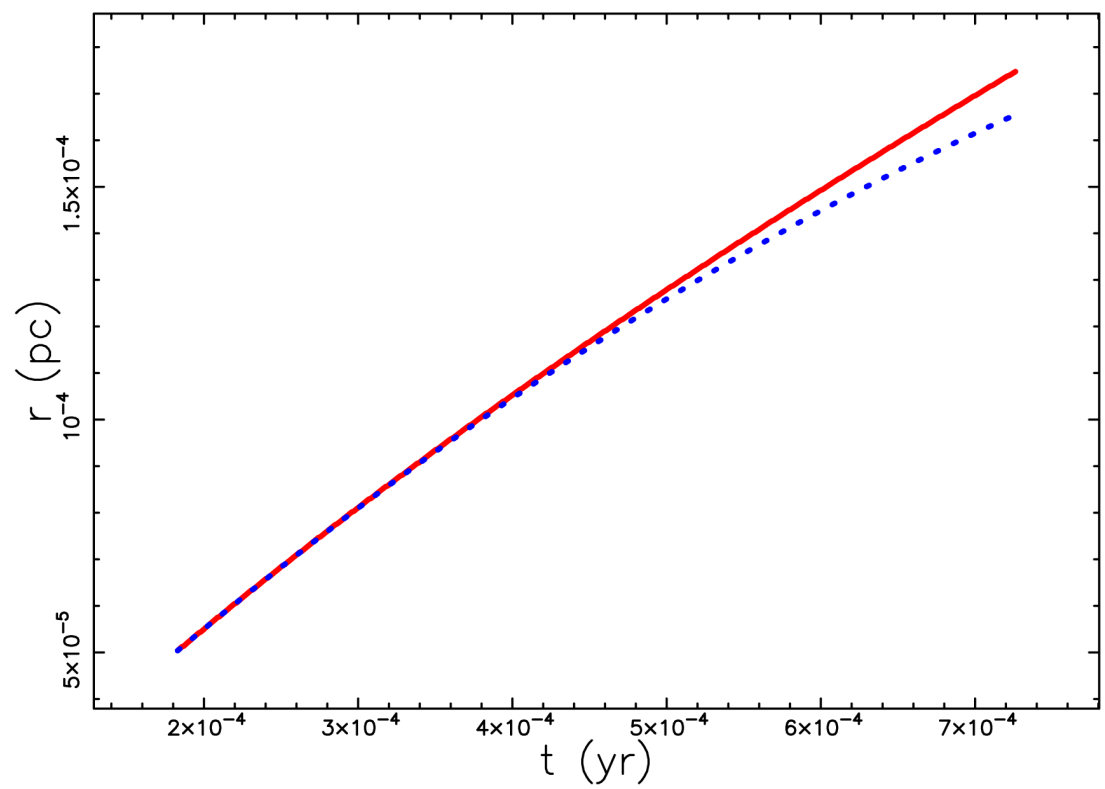

Figure 5. Numerical solution (full red line) and Taylor approximation (blue dashed line) for the NCD case with parameters as in Table 1 . The model is the conservation of the relativistic energy in the NCD case.

\section{Astrophysical Results}

We introduce one SN and two GRBs which were processed.

\subsection{The Case of SN 1993J}

In this subsection we adopt a classical equation of motion with a power law pro- 
file of density, see Section 4 . Figure 6 presents the decay of the $R$ magnitude of SN 1993J, which is type IIb, as well our theoretical curve.

We present the $H-\alpha$ with soft and hard band X-ray luminosities as well the theoretical luminosity in Figure 7. Figure 8 presents the radio flux density of SN

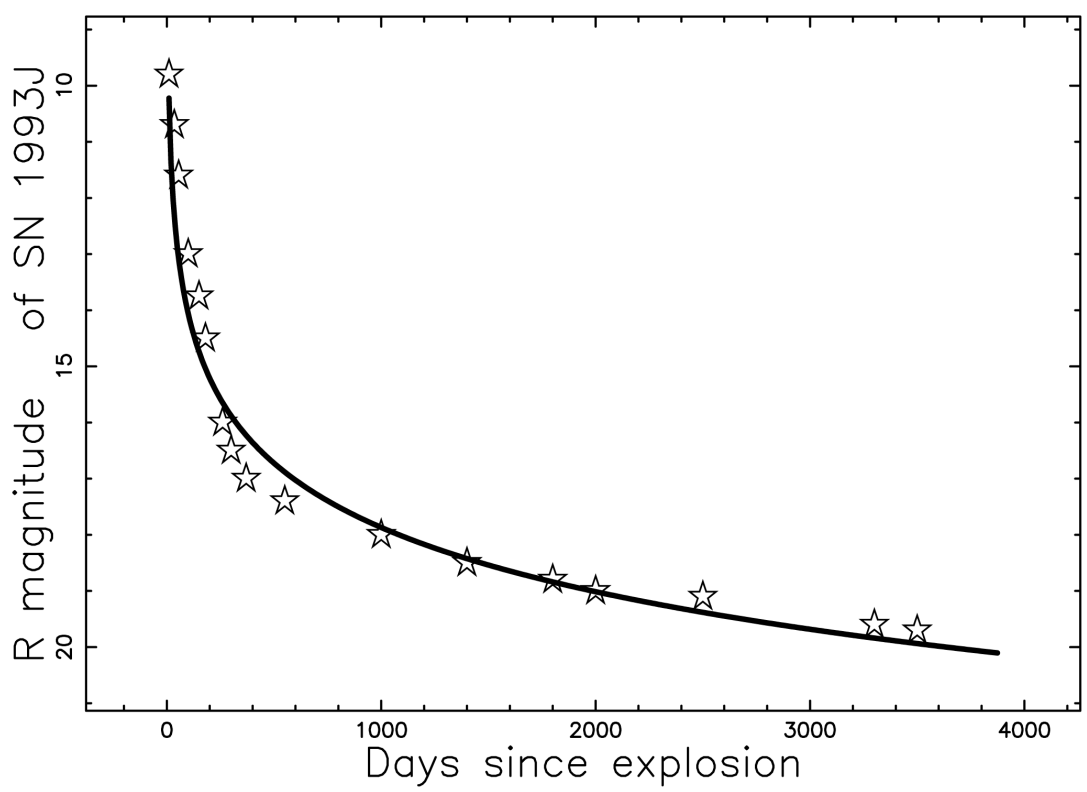

Figure 6. The $R$ LC of SN 1993J over $10 \mathrm{yr}$ (empty stars) and theoretical curve in the classical framework of a power law profile for the density as given by Equation (27) (full line). Parameters of the trajectory as in Table $1, d=6, k_{o b s}=-11.5$ and $\rho_{0}=1$. The data were extracted by the author from Figure 5 in Zhang et al. (2004).

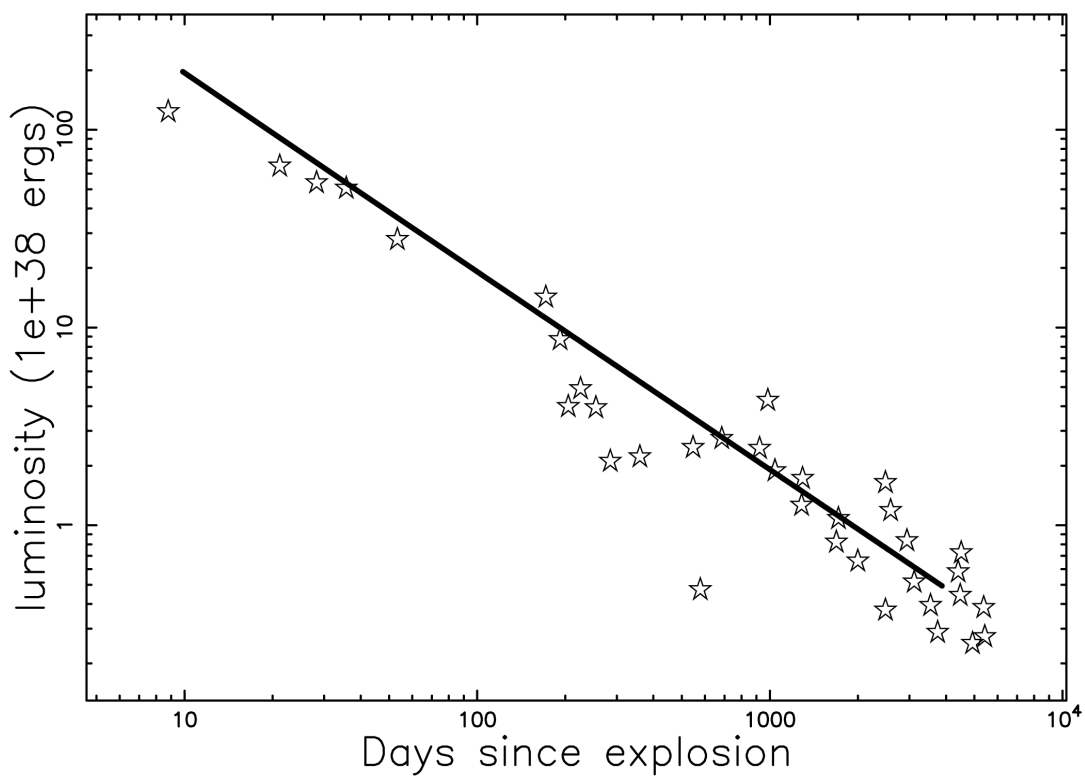

Figure 7. The $H-\alpha$ and the $2.0-8.0 \mathrm{keV}$ luminosities of SN 1993J over $10 \mathrm{yr}$ (empty stars) and theoretical curve in the classical framework of a power law profile for the density as given by Equation (28) (full line). Parameters of the trajectory as in Table $1, d=2.5$, $C_{\text {obs }}=1.5 \times 10^{17}$ and $\rho_{0}=1$. The data were extracted by the author from Figure 5 in [14]. 
1993J at $15.2 \mathrm{GHz}$ observed by the Ryle Telescope as well the theoretical flux, which requires a time dependent evaluation of the optical depth $\tau_{v}$, see Figure 9 .

Figure 10 presents the V-magnitude of SN 1993J for few days as well the theoretical magnitude and the time evolution of the optical depth $\tau_{v}$, see Figure 11 .

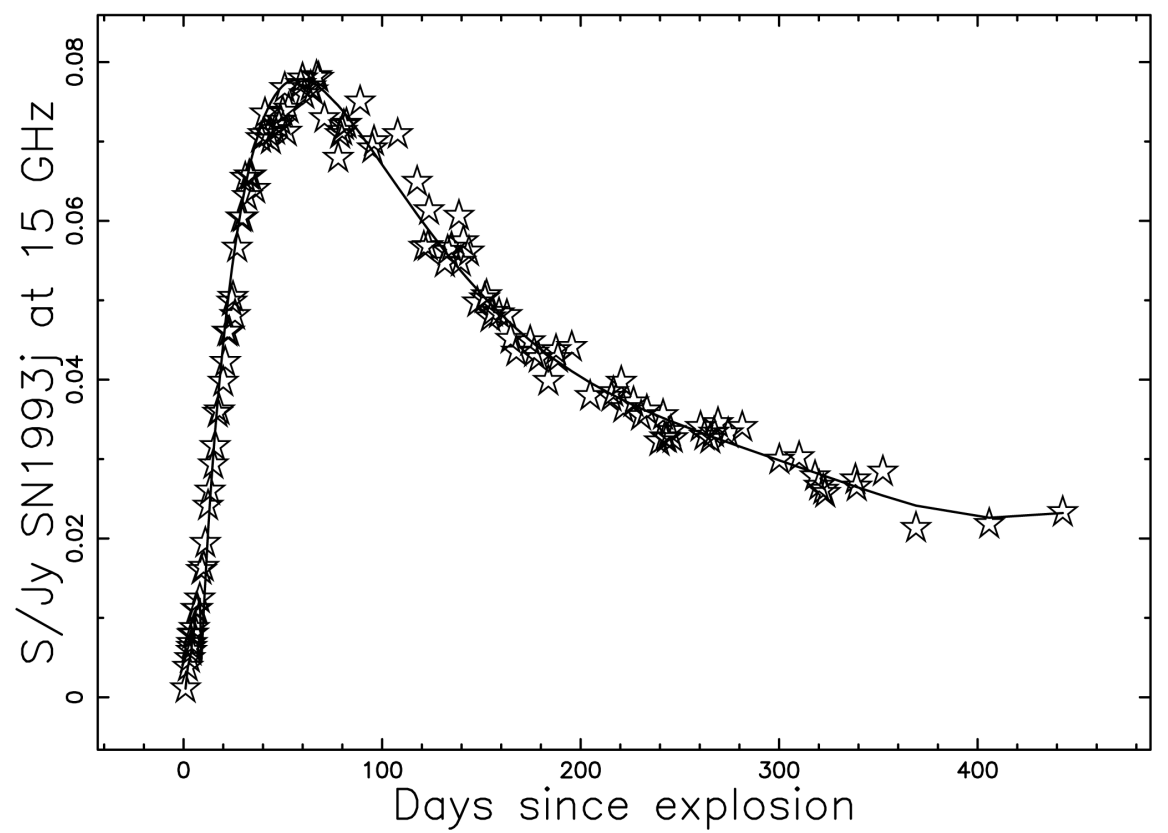

Figure 8. The radio flux density of SN 1993J over 443 days (empty stars) and theoretical behaviour in the classical framework of a power law profile for the density evaluated with formula (17) (full line). Parameters of the trajectory as in Table 1, $d=2.5, C_{o b s}=8.45 \times 10^{14}$ and $\rho_{0}=1$.

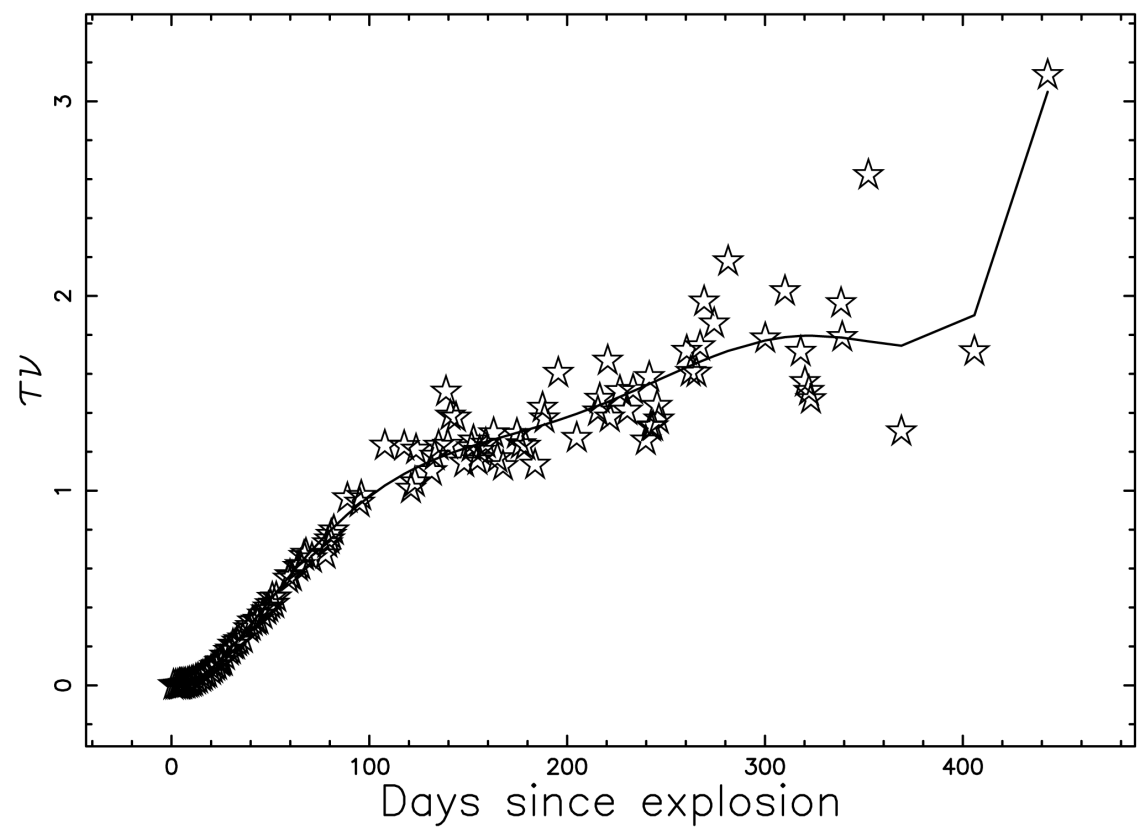

Figure 9. The time dependence of $\tau_{v}$ (empty stars) and a polynomial approximation of degree 6 (full line). Parameters as in Figure 8. 


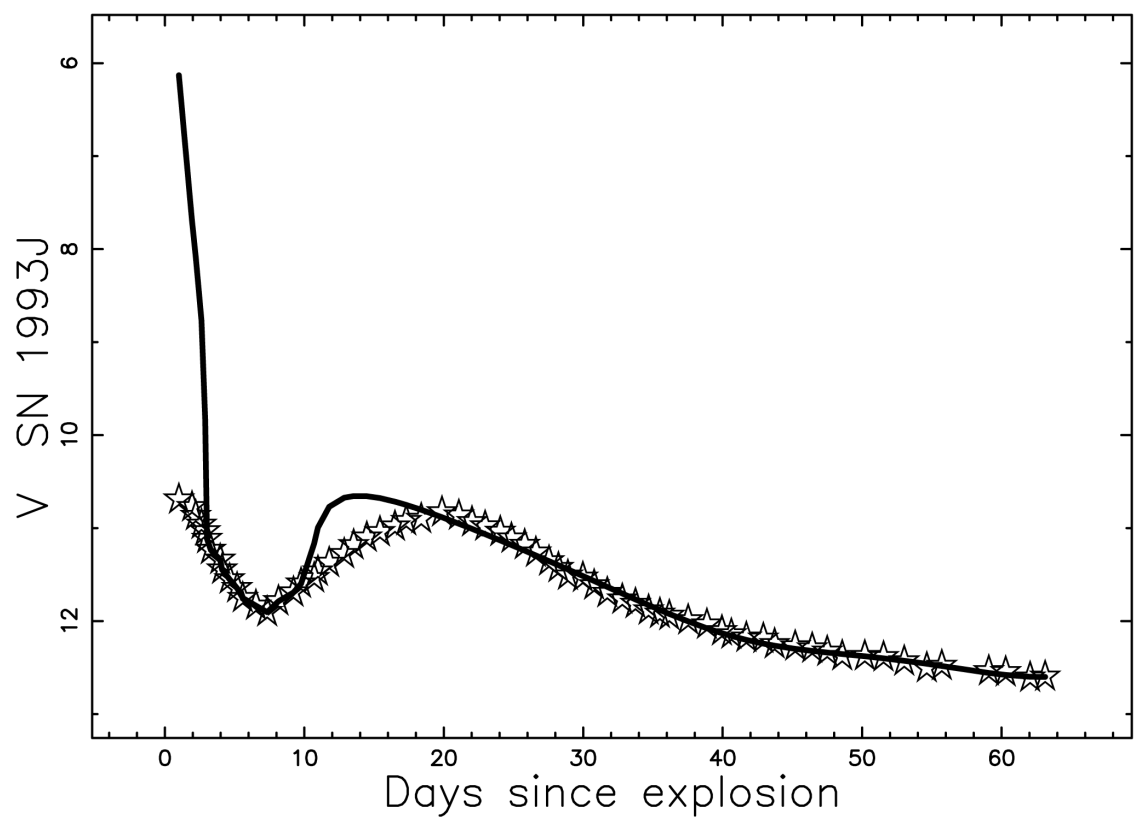

Figure 10. The $V$ LC of SN 1993J over 63 days (empty stars) and theoretical curve in the classical framework of a power law profile for the density as given by Equation (18) (full line). Parameters of the trajectory as in Table $1, d=6, k_{\text {obs }}=-12.5$ and $\rho_{0}=1$. The data were extracted by the author from Figure 4 in [13].

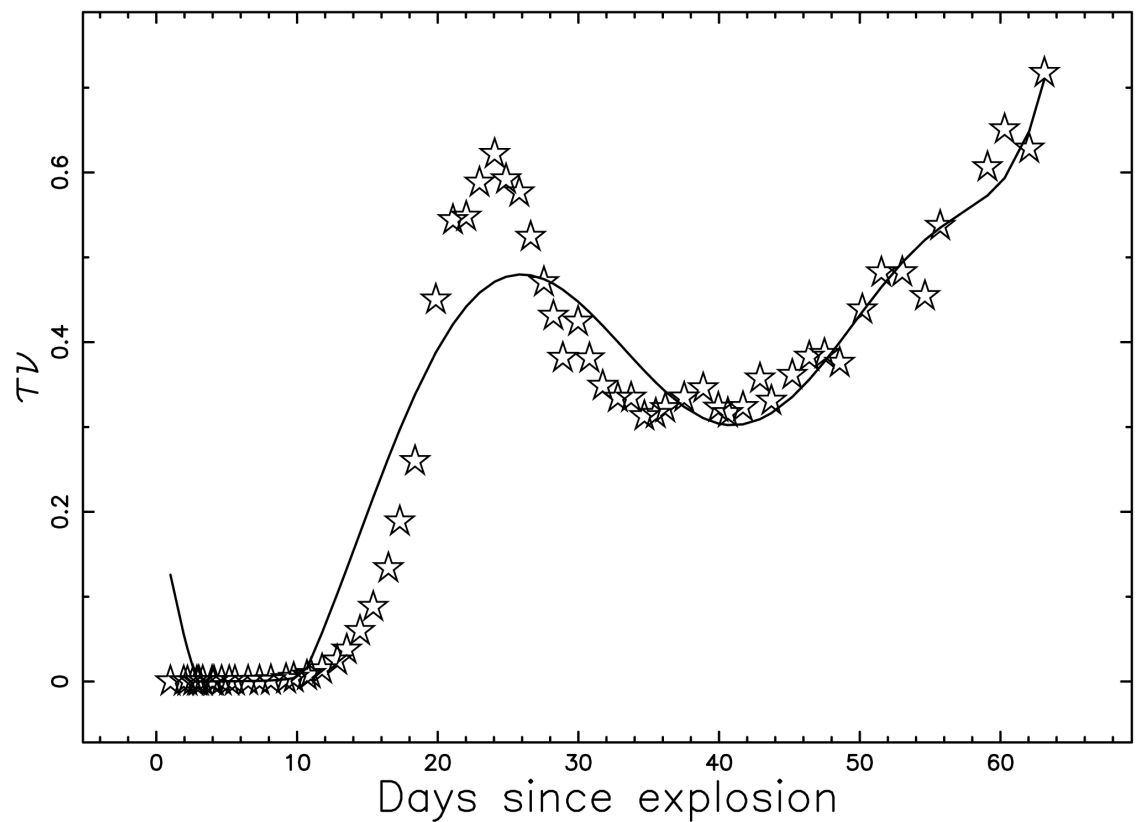

Figure 11. The time dependence of $\tau_{v}$ (empty stars) and a polynomial approximation of degree 10 (full line). Parameters as in Figure 10.

\subsection{The Case of GRB 050814}

In this subsection we adopt a relativistic equation of motion with an NFW profile for the density, see Section 5.1. Figure 12 presents the XRT flux of GRB 050814 and Figure 13 presents the temporal behaviour of the optical depth. 


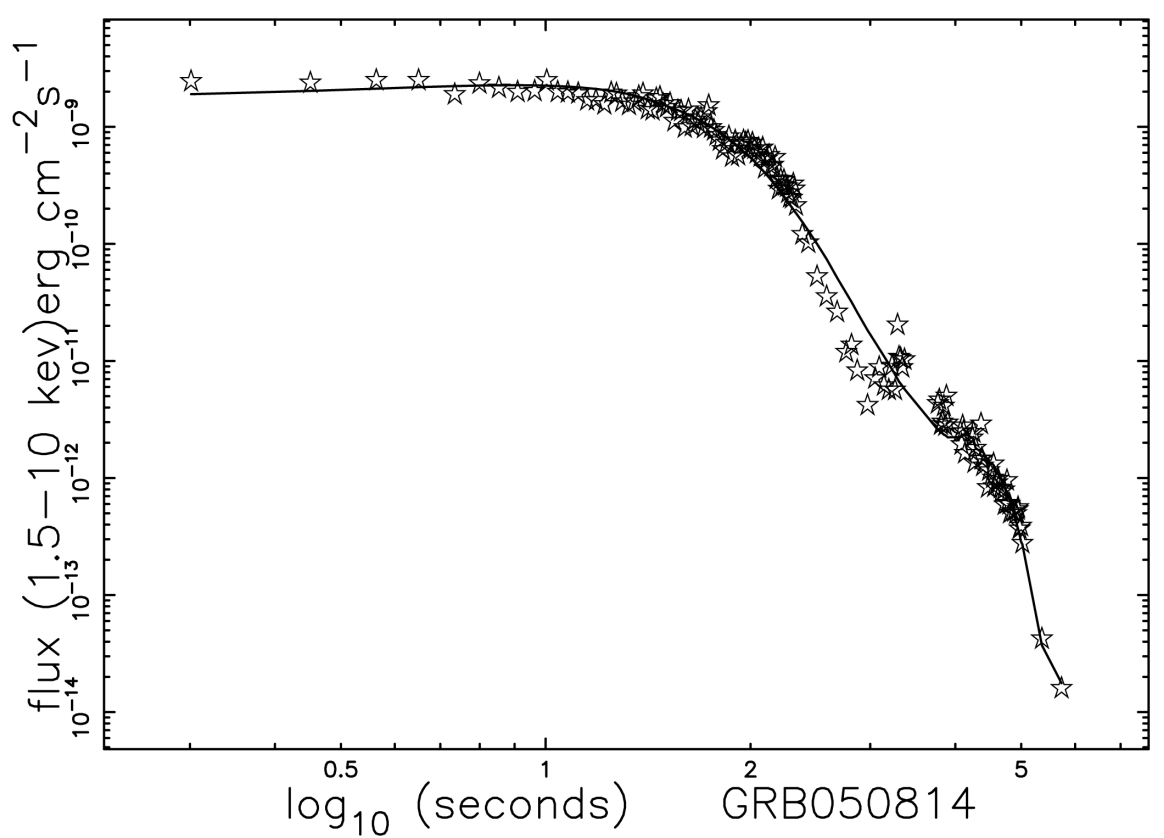

Figure 12. The XRT flux of GRB 050814 at 0.2 - $10 \mathrm{keV}$ (empty stars) and theoretical curve with velocity and radius as given by the NFW relativistic numerical model. The theoretical luminosity, which is corrected for absorption, is given by Equation (13) (full line).

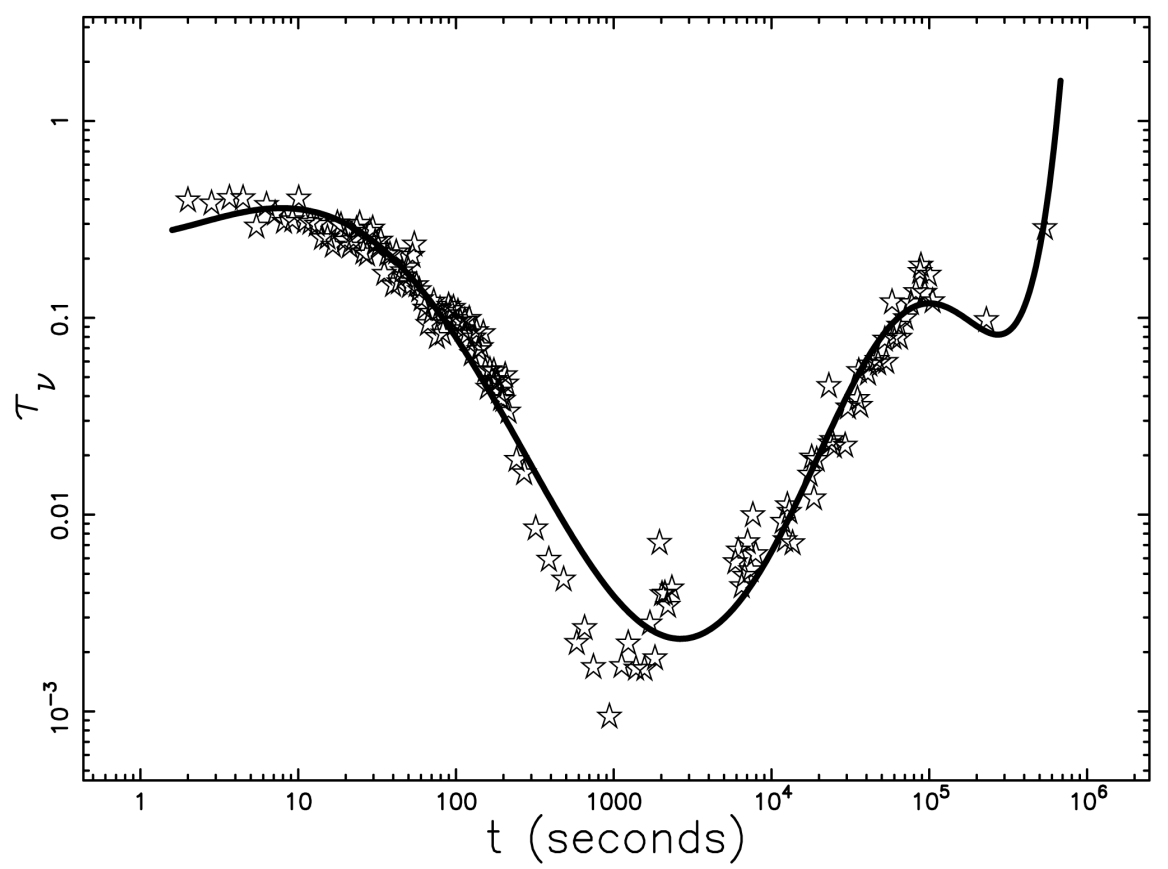

Figure 13. The time dependence of $\tau_{v}$ (empty stars) for GRB 050814 and a logarithmic polynomial approximation of degree 7 (full line). Parameters as in Figure 12.

\subsection{The Case of GRB 060729}

In this subsection we adopt a relativistic equation of motion for the NCD case, see Section 5.2. Figure 14 presents the LC of UVOT (U) apparent magnitude for GRB 060729 and Figure 15 presents the temporal behaviour of the optical depth. 


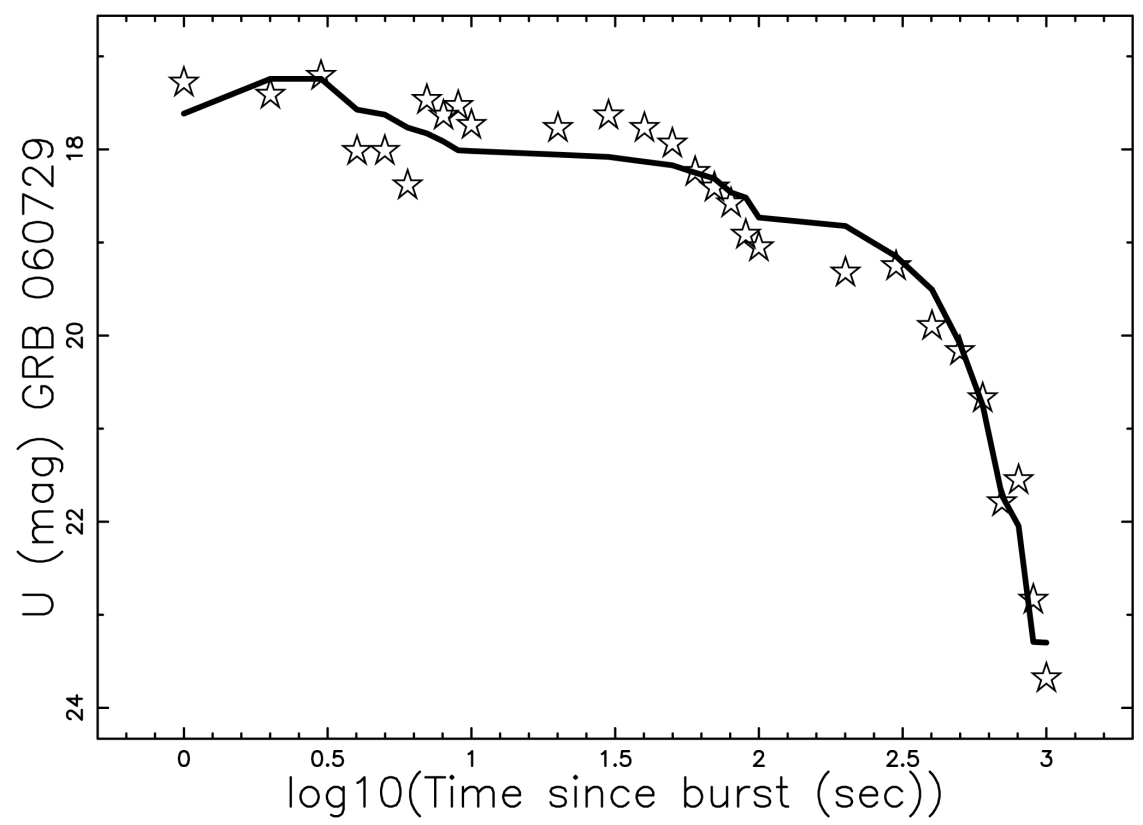

Figure 14. The LC of UVOT (U) + HST (F330W) for GRB 060729 (empty stars) and theoretical curve with radius as given by the NCD relativistic numerical model with data as in Table 1. The theoretical luminosity is given by Equation (13) (full line).

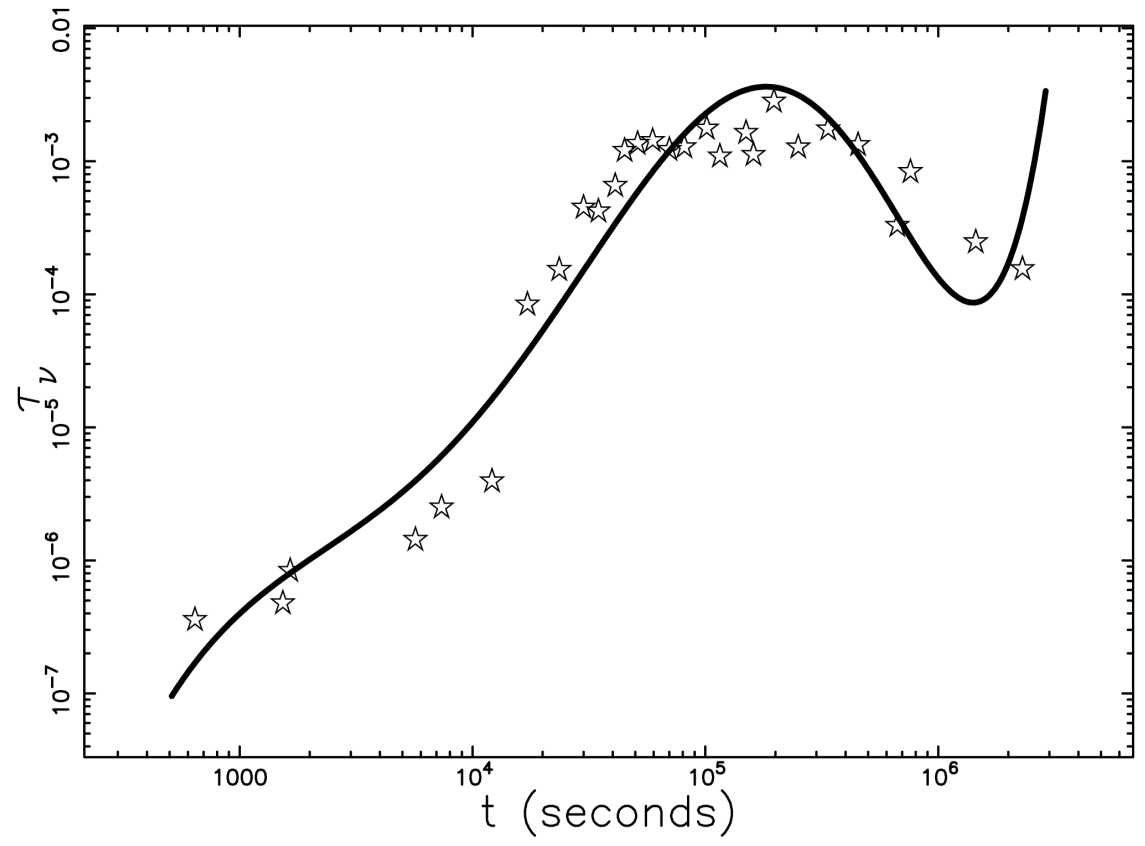

Figure 15. The time dependence of $\tau_{v}$ (empty stars) for GRB 060729 and a logarithmic polynomial approximation of degree 10 (full line). Parameters as in Figure 12.

\section{Acceleration and Magnetic Field}

The flux at frequency, $S_{v}$, in the radio band for SNs is parametrized by

$$
S_{v}=C_{v} v^{-\alpha_{r}},
$$

where $\alpha_{r}$ is the observed spectral index and $C_{v}$ is a constant. As a conse- 
quence, the luminosity, $L_{v}$, is

$$
L_{v}=4 \pi D^{2} S_{v},
$$

where $D$ is the distance. We now explain how it is possible to derive the magnetic field from the luminosity. The magnetic field for which the total energy of a radio source has a minimum is

$$
H^{\min }=1.5368 \frac{C_{12}^{2 / 7} L^{2 / 7}(1.0+k)^{2 / 7}}{\Phi^{2 / 7} R^{6 / 7}} \text { gauss, }
$$

where

$$
c_{12}=2 \frac{\sqrt{c_{1}}\left(-1+\alpha_{r}\right)\left(\sqrt{\nu_{1}} v_{2}^{\alpha_{r}}-v_{1}^{\alpha_{r}} \sqrt{v_{2}}\right)}{c_{2}\left(v_{1} v_{2}^{\alpha_{r}}-v_{2} v_{1}^{\alpha_{r}}\right)\left(-1+2 \alpha_{r}\right)},
$$

where $\alpha_{r}$ is the spectral index, $c_{1}$ and $c_{2}$ are two constants, $v_{1}$ and $v_{2}$ are the lower and upper frequency of synchrotron emission, $L$ is the luminosity in $\operatorname{ergs}^{-1}, k$ is the ratio between energy in heavy particle and electron energy, $\Phi$ is the fraction of source's volume occupied by the relativistic electrons and the magnetic field, and $R$ is the radius of the source; for more details see formula (7.14) in [21] or formula (5.109) in [27]. The constant $C_{12}$ is numerically evaluated in Table 8 of [21] and an example is presented in Figure 16. The scaling of the magnetic of equipartition as given by Equation (40) is

$$
H^{\min } \propto \frac{L^{2 / 7}}{R^{6 / 7}} .
$$

The first example presents the temporal behaviour of $H^{\min }$ for GRB 050814 in which we inserted the theoretical luminosity corrected for absorption, see Figure 17.

The second example presents the temporal behaviour of $H^{\text {min }}$ for SN 1993J in which we inserted the theoretical luminosity as given by the power law fit, see Figure 18.

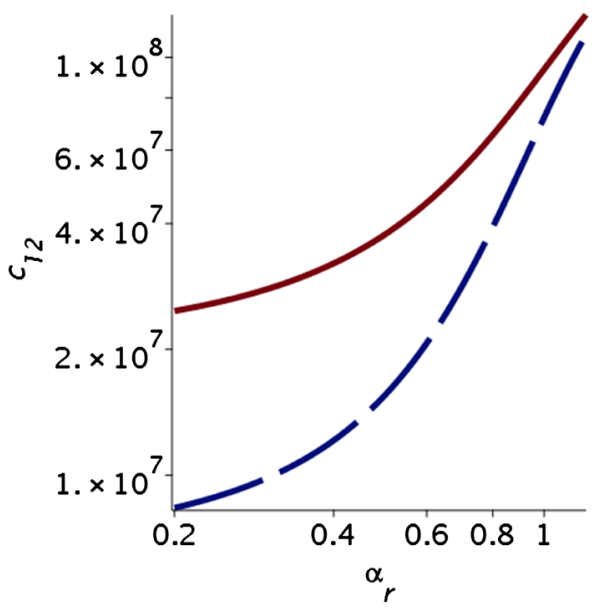

Figure 16. The constant $c_{12}$ as a function of the spectral index $\alpha_{r}$ when $v_{1}=10^{7} \mathrm{~Hz}$, $v_{2}=10^{10} \mathrm{~Hz}$ (red full line) and $v_{2}=10^{11} \mathrm{~Hz}$ (blue dashed line). 


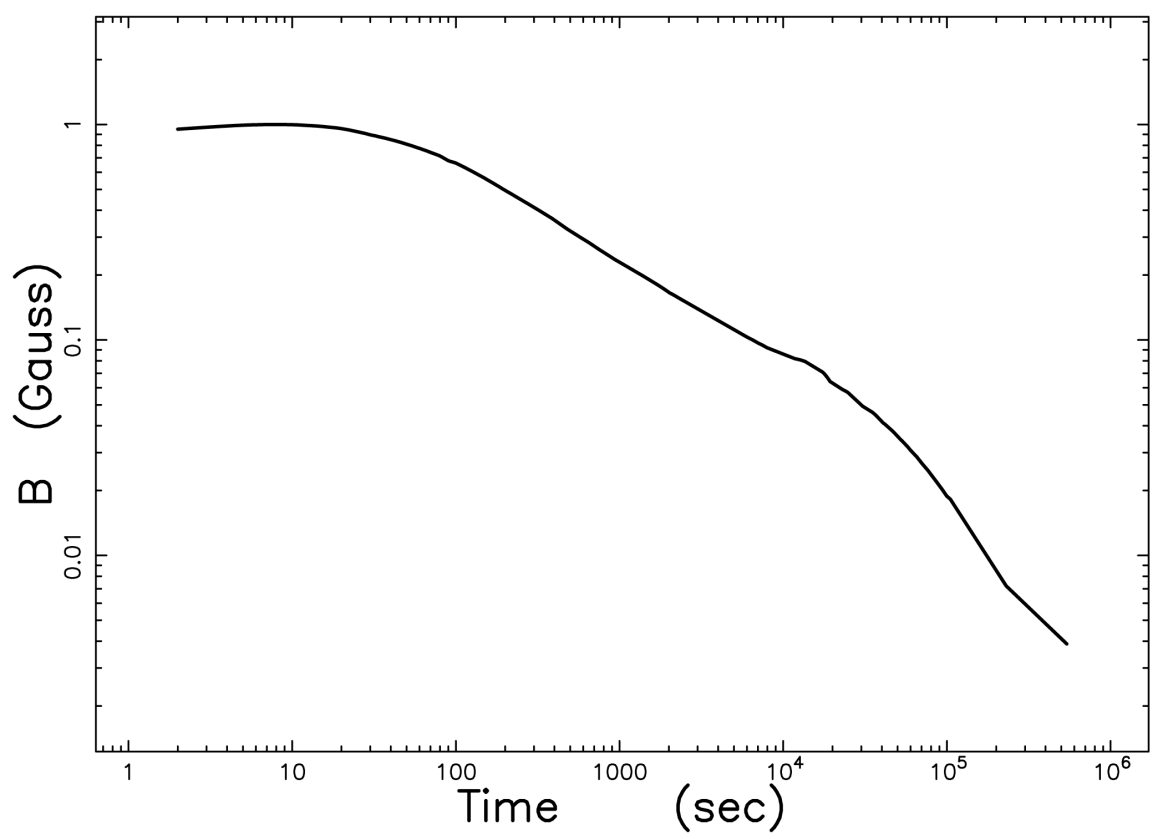

Figure 17. The time dependence (seconds) of the minimum magnetic field for GRB 050814 with theoretical luminosity as in Figure 13 when $H^{\min }=1$ gauss at $t=t_{0}$. The model is a fit to a power law.

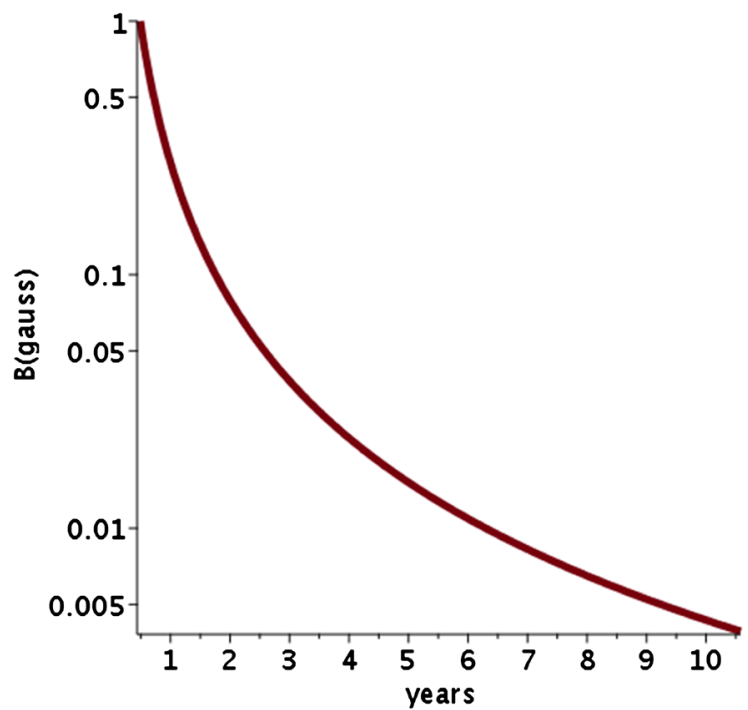

Figure 18. The time dependence (years) of the minimum magnetic field for SN 1993J with theoretical luminosity as given by formula (7) when $H^{\text {min }}=1$ gauss at $t=t_{0}$. The model is a fit to a power law.

An electron which loses its energy due to the synchrotron radiation has a lifetime of

$$
\tau_{r} \approx \frac{E}{P_{r}} \approx 500 E^{-1} H^{-2} \text { sec, }
$$

where $E$ is the energy in ergs, $H$ the magnetic field in Gauss, and $P_{r}$ is the total radiated power, see Eq. 1.157 in [28]. The energy is connected to the critical fre- 
quency, see Eq. 1.154 in [28], by

$$
v_{c}=6.266 \times 10^{18} \mathrm{HE}^{2} \mathrm{~Hz} .
$$

The lifetime for synchrotron losses is

$$
\tau_{\text {syn }}=39660 \frac{1}{H \sqrt{H v}} \mathrm{yr} .
$$

Following [29] [30], the gain in energy in a continuous form is proportional to its energy, $E$,

$$
\frac{\mathrm{d} E}{\mathrm{~d} t}=\frac{E}{\tau_{I I}},
$$

where $\tau_{I I}$ is the typical time scale,

$$
\frac{1}{\tau_{I I}}=\frac{4}{3}\left(\frac{u^{2}}{c^{2}}\right)\left(\frac{c}{L_{I I}}\right),
$$

where $u$ is the velocity of the accelerating cloud belonging to the advancing shell of the GRB, $c$ is the speed of light and $L_{I I}$ is the mean free path between clouds, see Eq. 4.439 in [28]. The mean free path between the accelerating clouds in the Fermi II mechanism can be found from the following inequality in time:

$$
\tau_{I I}<\tau_{\text {sync }},
$$

which corresponds to the following inequality for the mean free path between scatterers

$$
L<16182.11 \frac{u^{2}}{H^{3 / 2} \sqrt{v} c^{2}} \text { pc. }
$$

The mean free path length for a GRB which emits synchrotron emission around $1 \mathrm{keV}\left(2.417 \times 10^{17} \mathrm{~Hz}\right)$ is

$$
L<3.2908 \times 10^{-5} \frac{\beta^{2}}{H^{3 / 2} \sqrt{E(\mathrm{keV})}} \mathrm{pc}
$$

where $\beta$ is the velocity of the cloud divided by the speed of light. When this inequality is fulfilled, the direct conversion of the rate of kinetic energy into radiation can be adopted. Figure 19 presents the above line in the framework of the fitted model. The mean free path length varies from $1.5 \times 10^{-5}$ to $1.5 \times 10^{-4}$ with respect to the numerical value of the advancing radius.

\section{Conclusions}

\section{Classical and relativistic flux of energy:}

The classical flux of kinetic energy has an analytical expression in the case of energy conservation in the presence of a power law profile for the density, see Equation (26). The relativistic flux of energy in the two cases here analysed can only be found numerically.

\section{Momentum versus energy:}

The comparison of the trajectories for SN 1993J for the four possibilities, classic or relativistic, conservation of energy or momentum, is presented in Table 2. 


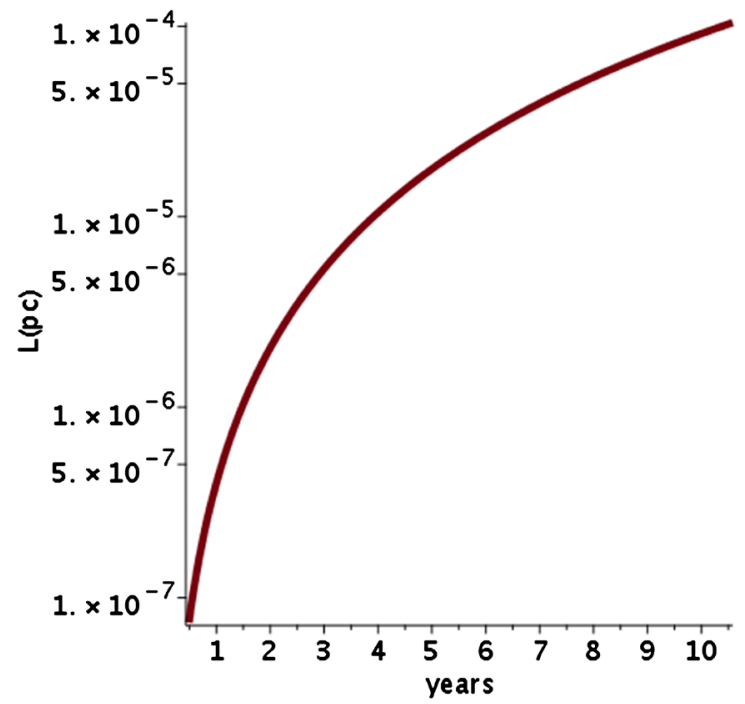

Figure 19. The time dependence (years) of the mean free path length for SN 1993J when $E=1 \mathrm{keV}$. The model is a fit to a power law.

Table 2. Type of regime, conservation, model, $\chi^{2}$ and reference for SN 1993J.

\begin{tabular}{ccccc}
\hline regime & conservation & model & $\chi^{2}$ & Reference \\
\hline classical & momentum & inverse power law & 276 & Figure 6 in [31] \\
classical & momentum & Plummer profile & 265 & Figure 8 in [32] \\
relativistic & momentum & Lane-Emden profile & 471 & Figure 10 in [32] \\
relativistic & energy & power law profile & 6387 & Figure 4 in [25] \\
relativistic & energy & exponential profile & 13,145 & Figure 6 in [25] \\
relativistic & energy & Emden profile & 8888 & Figure 8 in [25] \\
classical & energy & power law profile & 176.6 & Figure 1 in this paper \\
relativistic & energy & NFW profile & 823 & Figure 2 in this paper \\
relativistic & energy & NCD & 9589 & Figure 4 in this paper \\
\hline
\end{tabular}

The best results are obtained for the energy conservation in the presence of a power law profile in the present paper, see Equation (24).

\section{Light curve:}

The luminosity in the various astronomical bands is here assumed to be proportional to the classical or relativistic flux of mechanical kinetic energy. This theoretical dependence is not enough and the concept of optical depth should be introduced. Due to the complexity of the time dependence of the optical depth, a polynomial approximation of degree $M$ with time as independent variable has been suggested, see Equation (20) which is used in a linear or logarithmic form.

Comparison with astronomical data:

The framework of conversion of the classical flux of mechanical kinetic energy into the various astronomical bands coupled with a time dependence for the optical depth allowed simulating the various morphologies of the LC of supernovae. In particular, in the case of SN 1993J we modeled: (i) the $R$ LC of SN 
1993J over $10 \mathrm{yr}$, see Figure 6, (ii) the $H-\alpha$ and the $2.0-8.0 \mathrm{keV}$ luminosities over $10 \mathrm{yr}$, see Figure 7, (iii) the radio flux density over 443 days, see Figure 8 and (iv) $V$ LC over 63 days, see the double peak visible in Figure 10. The LC of of GRB 050814 at $0.2-10 \mathrm{keV}$ was modeled in Figure 12 and that of UVOT (U) + HST (F330W) for GRB 060729 was modeled in Figure 14.

\section{Magnetic field}

The minimum magnetic field depends on the luminosity and this allows to derive its theoretical dependence on time, see Figure 18. The above dependence allows deriving the distance for the mean free path between accelerating clouds for the Fermi II mechanism when the relativistic electron emits synchrotron radiation in the keV region, see Figure 19.

\section{Conflicts of Interest}

The author declares no conflicts of interest regarding the publication of this paper.

\section{References}

[1] Dimitriadis, G., Foley, R.J., Rest, A., et al. (2019) K2 Observations of SN 2018oh Reveal a Two-Component Rising Light Curve for a Type Ia Supernova. ApJ Letters, 870, L1.

[2] Palliyaguru, N.T., Corsi, A., Frail, D.A., et al. (2019) The Double-Peaked Radio Light Curve of Supernova PTF11qcj. Astrophysical Journal, 872, Article No. 201. https://doi.org/10.3847/1538-4357/aaf64d

[3] Papadogiannakis, S., Goobar, A., Amanullah, R., et al. (2019) R-Band Light-Curve Properties of Type Ia Supernovae from the (Intermediate) Palomar Transient Factory. Monthly Notices of the Royal Astronomical Society, 483, 5045-5076. https://doi.org/10.1093/mnras/sty3301

[4] Wang, S.Q., Cano, Z., Li, L., et al. (2019) Modeling the Light Curves of the Luminous Type Ic Supernova 2007D. Astrophysical Journal, 877, 20. https://doi.org/10.3847/1538-4357/ab1903

[5] Ricks, W. and Dwarkadas, V.V. (2019) Excavating the Explosion and Progenitor Properties of Type IIP Supernovae via Modeling of Their Optical Light Curves. Astrophysical Journal, 880, 59. https://doi.org/10.3847/1538-4357/ab287c

[6] Tsuna, D., Kashiyama, K., Shigeyama, T., et al. (2019) Type IIn Supernova Light Curves Powered by Forward and Reverse Shocks. Astrophysical Journal, 884, 87. https://doi.org/10.3847/1538-4357/ab40ba

[7] Kushnir, D. and Waxman, E. (2020) Constraints on the Density Distribution of Type Ia Supernovae Ejecta Inferred from Late-Time Light-Curve Flattening. Monthly Notices of the Royal Astronomical Society, 493, 5617. https://doi.org/10.1093/mnras/staa690

[8] Koo, H., Shafieloo, A., Keeley, R.E., et al. (2020) Model-Independent Constraints on Type Ia Supernova Light-Curve Hyperparameters and Reconstructions of the Expansion History of the Universe. Astrophysical Journal, 899, 9. https://doi.org/10.3847/1538-4357/ab9c9a

[9] Wang, S.Q. and Li, L. (2020) Exploring the Energy Sources Powering the Light Curve of the Type Ibn Supernova PS15dpn and the Mass-Loss History of the SN Progenitor. Astrophysical Journal, 900, 83. 
https://doi.org/10.3847/1538-4357/aba6e9

[10] Marcaide, J.M., Mart-Vidal, I., Alberdi, A. and Pérez-Torres, M.A. (2009) A Decade of SN 1993J: Discovery of Radio Wavelength Effects in the Expansion Rate. $A \& A$, 505, 927. https://doi.org/10.1051/0004-6361/200912133

[11] Zaninetti, L. (2014) The Physics of the Optical Light Curve in Supernovae. Applied Physics Research, 6, 118. https://doi.org/10.5539/apr.v6n2p118

[12] Zhang, T., Wang, X., Zhou, X., Li, W., Ma, J., Jiang, Z. and Li, Z. (2004) Optical Photometry of SN 1993J: 1995 to 2003. The Astronomical Journal, 128, 1857. https://doi.org/10.1086/423699

[13] Benson, P.J., Herbst, W., Salzer, J.J., Vinton, G., Hanson, G.J., Ratcliff, S.J., Winkler, P.F., Elmegreen, D.M., Chromey, F., Strom, C., Balonek, T.J. and Elmegreen, B.G. (1994) Light Curves of SN 1993J from the Keck Northeast Astronomy Consortium. The Astronomical Journal, 107, 1453. https://doi.org/10.1086/116958

[14] Chandra, P., Dwarkadas, V.V., Ray, A., Immler, S. and Pooley, D. (2009) X-Rays from the Explosion Site: 15 Years of Light Curves of SN 1993J. Astrophysical Journal, 699, 388. https://doi.org/10.1088/0004-637X/699/1/388

[15] Pooley, G.G. and Green, D.A. (1993) Ryle Telescope Observations of Supernova 1993J at 15-GHZ-The First 115 Days. Monthly Notices of the Royal Astronomical Society, 264, L17. https://doi.org/10.1093/mnras/264.1.L17

[16] Jakobsson, P., Levan, A. and Fynbo, J.P. (2006) A Mean Redshift of 2.8 for Swift Gamma-Ray Bursts. $A$ \& A, 447, 897. https://doi.org/10.1051/0004-6361:20054287

[17] Cano, Z., Bersier, D., Guidorzi, C., et al. (2011) A Tale of Two GRB-SNe at a Common Redshift of $z=0.54$. Monthly Notices of the Royal Astronomical Society, 413, 669 .

[18] Nagy, A.P., Ordasi, A., Vinkó, J. and Wheeler, J.C. (2014) A Semianalytical Light Curve Model and Its Application to Type IIP Supernovae. $A \& A, 571, \mathrm{~A} 77$. https://doi.org/10.1051/0004-6361/201424237

[19] Zaninetti, L. (2015) Relativistic Scaling Laws for the Light Curve in Supernovae. Applied Physics Research, 6, 48. https://doi.org/10.5539/apr.v7n3p48

[20] Longair, M.S. (2011) High Energy Astrophysics III. Cambridge University Press, Cambridge.

[21] Pacholczyk, A.G. (1970) Radio Astrophysics. Nonthermal Processes in Galactic and Extragalactic Sources. Freeman, San Francisco.

[22] Rybicki, G. and Lightman, A. (1991) Radiative Processes in Astrophysics. Wiley Interscience, New York.

[23] Press, W.H., Teukolsky, S.A., Vetterling, W.T. and Flannery, B.P. (1992) Numerical Recipes in FORTRAN. The Art of Scientific Computing. Cambridge University Press, Cambridge.

[24] Zaninetti, L. (2020) Energy Conservation in the Thin Layer Approximation: I. The Spherical Classic Case for Supernovae Remnants. International Journal of Astronomy and Astrophysics, 10, 71. https://doi.org/10.4236/ijaa.2020.102006

[25] Zaninetti, L. (2020) Energy Conservation in the Thin Layer Approximation: III. The Spherical Relativistic Case for Supernovae. International Journal of Astronomy and Astrophysics, 10, 285-301.

[26] Navarro, J.F., Frenk, C.S. and White, S.D.M. (1996) The Structure of Cold Dark Matter Halos. Astrophysical Journal, 462, 563. https://doi.org/10.1086/177173

[27] Condon, J.J. and Ransom, S.M. (2016) Essential Radio Astronomy. Princeton Uni- 
versity Press, Princeton. https://doi.org/10.1515/9781400881161

[28] Lang, K.R. (1999) Astrophysical Formulae. Third Edition, Springer, New York.

[29] Fermi, E. (1949) On the Origin of the Cosmic Radiation. Physical Review, 75, 1169. https://doi.org/10.1103/PhysRev.75.1169

[30] Fermi, E. (1954) Galactic Magnetic Fields and the Origin of Cosmic Radiation. Astrophysical Journal, 119, 1. https://doi.org/10.1086/145789

[31] Zaninetti, L. (2011) Time-Dependent Models for a Decade of SN 1993J. Astrophysics and Space Science, 333, 99. https://doi.org/10.1007/s10509-011-0609-x

[32] Zaninetti, L. (2014) A Classical and a Relativistic Law of Motion for Spherical Supernovae. Astrophysical Journal, 795, 80.

https://doi.org/10.1088/0004-637X/795/1/80 